\title{
AN ITERATIVE APPROACH TO IMAGE SUPER-RESOLUTION
}

\author{
Vivek Bannore' and Leszek Swierkowski ${ }^{2}$ \\ 'School of Electrical and Information Engineering, University of South Australia, Mawson \\ Lakes SA 5095,Australia. Phone; +61-4-05149738, Vivek Bamores postsrads anistsedu.au \\ ${ }^{2}$ Defence Science and Technology Organisation, Edinburgh SA 51Il, Austrulia.
}

\begin{abstract}
Undersampling and aliasing occurs frequently in many imaging systems leading to degradation of image quality. Super-resolution attempts to reconstruct a high-resolution image by fusing the incomplete scene information contained in the sequence of under-sampled images. This paper investigates iterative approaches to super-resolution. We propose algorithm that utilises a relatively small number of low-resolution images and is computationally inexpensive. Experimental results of reconstruction are presented.
\end{abstract}

Key words: Image processing, Super-Resolution, Iterative technique, Inage Interpolation 


\section{INTRODUCTION}

In many applications such as satellite imaging, remote sensing, forensic science and computer vision applications high-resolution imagery is essential. Despite the recent advances in sensor technology the requirements for high quality imagery very often exceed the capabilities of imaging systems. In such cases it is desirable to develop image processing techniques that enhance the resolution of images. A promising technique of superresolution attempts to reconstruct a high-quality image from a number of undersampled images of the same scene.

The super-resolution technique takes advantage of the existence of relative motion between the sensor and the scene during the acquisition process. In the presence of such motion each low-resolution image frame acquired by the imaging system carries slightly different information about the scene. Super-resolution attempts to fuse this information during the image reconstruction process.

High-resolution image reconstruction techniques have been researched in both the frequency and the spatial domains. In general, the current techniques are unable to maintain a balance between improving spatial resolution and keeping the computational time low. Consequently, there is a challenge to develop new, more efficient techniques for super-resolution. Our aim is to address the shortcomings of algorithms proposed in literature and to explore the possibility of developing techniques that would be able to reconstruct high-resolution images without excessive computational cost.

In this paper we present a simple, iterative technique for super-resolution that is computationally inexpensive without sacrificing quality of the reconstructed image. The paper is organized as follows. In the next section a brief review of the existing super-resolution techniques is given. Section 3 describes image interpolation based super-resolution. The implementation of our algorithm is described in Section 4, and Section 5 describes iterative improvement technique. Finally, Section 6 presents our preliminary results of high-resolution image reconstruction.

\section{EXISTING SUPER-RESOLUTION TECHNIQUES}

A variety of approaches can be found in the literature for the reconstruction of high-resolution image by fusing data from a series of lowresolution frames. For the purpose of this paper a brief description of existing techniques is presented in this section. More comprehensive overviews can be found in $[1-3]$. 
The super-resolution model $[1,3-7]$ is based on a sequence of $N$ lowresolution (LR) images $\underline{b}_{K}(K=1 \ldots N)$ of the same scene. The scene is represented by a single high-resolution (HR) image $X$ that we wish to reconstruct. Each measured LR image is the result of sampling of the ideal high-resolution real scene and is subjected to camera and atmospheric blur, motion effects, geometrical warping and decimation. It is assumed that a linear operator $A_{K}$ represents all these imaging factors and that the LR images are contaminated by an additive Gaussian noise $\mathrm{E}$. Therefore, the super-resolution model is represented by the following equation:

$$
\underline{b}_{K}=A_{K} \underline{X}+\underline{E},
$$

where the images are represented as vectors (shown by an underscore), ordered column-wise lexicographically. The problem of super-resolution is then solved, in the least-squares sense, by minimization of the error $\tau$ between the actually measured and the predicted LR images:

$$
\tau=\sum_{K=1}^{N}\left[\underline{b}_{K}-A_{K} \underline{X}\right]^{2}
$$

The estimation of the HR image from the equation (2) is known to be an ill-conditioned problem, in the sense that small perturbations of LR images can lead to large changes in the reconstructed image. As a result the reconstruction problem is intrinsically unstable. A commonly used procedure that alleviates this difficulty is adding a Regularization term to equation (2):

$$
\tau=\sum_{K=1}^{N}\left[\underline{b}_{K}-A_{K} \underline{X}\right]^{2}+\lambda[Q \underline{X}]^{2} .
$$

$Q$ represents here a stabilization matrix and $\lambda>0$ is the regularization parameter. The regularization term can incorporate some a priori knowledge about the real high-resolution scene (smoothness, for example) and has to be chosen in such a way as to ensure that the reconstruction $X$ is stable with respect to small variations in the image data.

Most published techniques attempt to solve equation (2) or its regularized version (3) for the case where image motion is characterized by a global translation or rotation $[8,9]$. Hardie et al [8] used steepest descent and conjugate-gradient techniques for minimizing the cost function eq. (3) with a simple regularization term that enforced the smoothness of the solution. The 
iterative back-projection method was adopted in [9]. Tuinstra et al [10] proposed a method for treating non-global motion that used automated motion segmentation and registration.

The techniques based on optimization of the reconstructed image by minimization of the cost function (2) or (3) are usually applied in the spatial domain. In contrast, Tsai and Huang [11] were the first to propose the frequency domain approach for solving the super-resolution problem. This method directly addresses the removal of aliasing artifacts. The frequency domain approach is based on an assumption that the original high-resolution image is band-limited and exploits the translational property of the Fourier Transform. It makes use of the aliasing relationship between the Continuous Fourier Transform (CFT) of the original real scene and the Discrete Fourier Transform (DFT) of the observed low-resolution images.

In their paper, Tsai and Huang assumed the sequence of low-resolution frames to be free from distortions such as blur or noise. The computational cost of the method was found to be relatively low, but due to the limitation in geometric warping the approach did not gain much popularity.

In general, however, the problem of super-resolution is computationally expensive, ill-posed and underdetermined. In an attempt to alleviate the computational cost, Nguyen et al. $[12,13]$ introduced the concept of Preconditioning to the super-resolution problem. Preconditioning transforms the original system into one with the same solution but with better convergence properties. It therefore can be solved faster.

In this paper, we adopt a fast iterative approach for solving the problem of image super-resolution. In this method, a well-defined interpolation kernel is used to generate a first approximation to the reconstructed image. This approximation is then iteratively improved to produce the final highresolution solution. The paper compares the most commonly-used interpolation kernels and evaluates the efficiency of the reconstruction as a function of the number of LR images.

\section{IMAGE INTERPOLATION BASED SUPER- RESOLUTION}

It is well known result that the reconstruction $X$ in equation (3) tends to the generalized inverse solution as the regularization term vanishes $(\lambda \rightarrow 0)$. This result is the starting point for the method adopted in this paper. Although the method can be derived explicitly from the equation (3), for the purpose of this short communication we adopt more intuitive description, deferring mathematically rigorous derivation to a future publication. 
The method consists of several steps. At the initial stage the camera motion has to be taken into account. To accomplish this, a sequence of observed low-resolution images of the scene we want to super-tesolve is registered precisely relative to the reference $L R$ frame. Once this is achieved, a high-resolution image grid is populated with pixels from low-resolution images by placing them at the appropriate grid-points according to the registration information. Since the number of low-resolution images is limited, the whole composite grid template is not completely filled. It is worth mentioning at that point that under certain conditions this sparse image is related to the generalized inverse solution of equation (2).

The high-resolution image is finally estimated by interpolating the sparse grid to populate the empty pixels. This is an important step that has significant effect on the accuracy of the reconstruction. In an attempt to quantify the effect of interpolation on the reconstruction, the several image interpolation techniques were compared. Computer experiments were carried out using the following most commonly used interpolation methods:

a) Linear Interpolation

b) Nearest-Neighbor Interpolation

c) Truncated Sinc Interpolation

d) Lanczos 2 Windowed Sinc Interpolation

e) Lanczos 3 Windowed Sinc Interpolation

f) Cubic Spline Polynomial (Order 4 and 5)

g) Gaussian Interpolation (Order 2,6 and 10)

\section{IMPLEMENTATION}

The algorithm described briefly in the previous section was coded in MATLAB, making use of the Image Processing Toolbox. A number of highresolution images of sizes 512 by 512 and 1024 by 1024 were used for our testing purposes. In order to quantify the accuracy of the reconstruction a synthetic set of low-resolution images was generated for each HR image by applying random translations. The reconstruction procedure was then applied to the LR images and the reconstructed HR image was compared with the original. The interpolation methods listed above were implemented.

Two examples of the super-resolution reconstruction are presented here. Figure 1 shows LR images where only one image from each sequence is displayed. In these particular examples ten LR images were used for each reconstruction and the subsampling ratios were 16 and 12 for panels (a) and (b), respectively. The reconstructed HR images are shown in Figure 2. By inspecting the reconstructed images one can clearly see the deficiency of the reconstruction process. The periodic artifacts that are visible in the 
interpolated images are due to the high number of empty blocks in the composite high-resolution grid. These empty blocks appear in the HR grid as a result of irregular sampling of the scene caused by random movements between LR frames. The quality of the HR image increases significantly with the number of LR frames used in the reconstruction process. For practical reasons, however, it is important to keep the number of LR images as low as possible. Therefore, in the next section, we adopt an iterative approach to remove these visible artifacts and improve the estimated highresolution solution.

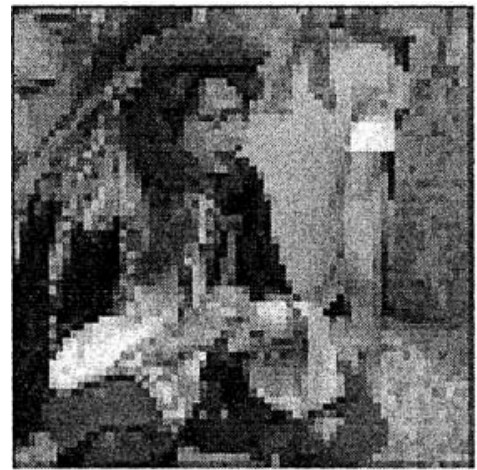

(a)

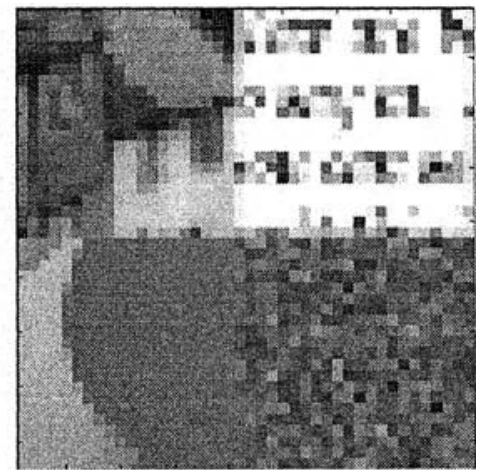

(b)

Figure 1. (a) One of the ten low-resolution images with sampling ratio of 16 . Size - [64 x 64$\}$. (b) One of the ten low-resolution images with sampling ratio of 12 . Size $-[42 \times 42\}$.

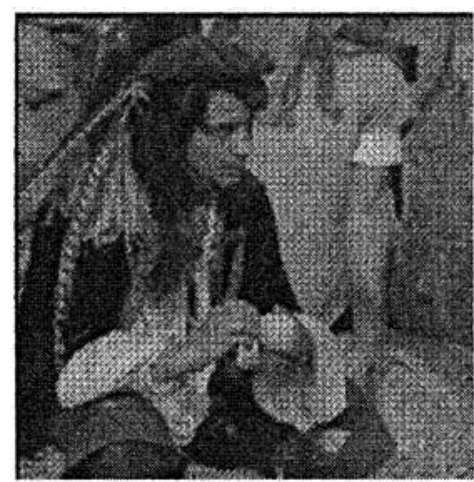

(a)

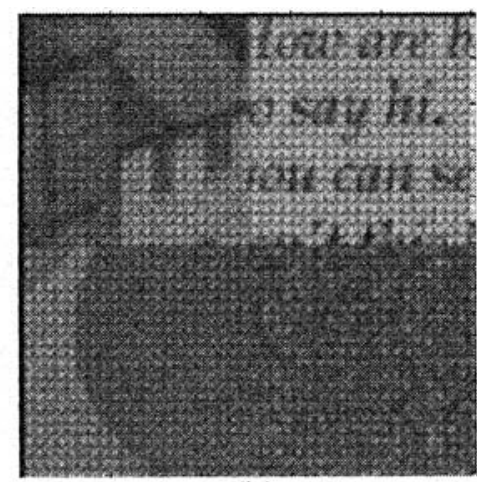

(b)

Figure 2. First Approximation interpolated images. (a) [1024 $\times 1024]$ (b) [504 x 504$]$ 


\section{ITERATIVE IMPROVEMENT TECHNIQUE}

A solution of the least-square problem eq. (3) can be sought by solving the equivalent normal equation, which involves the inversion of a linear operator. Suppose that only an approximate inverse is known, the solution can then be improved by an iterative approach described in [14].

In the context of super-resolution reconstruction, the approximate inverse operation is given by our interpolation-based reconstruction, as described in the previous sections. The interpolated HR image is fed into the iteration scheme as the first approximation image $X_{1}$. This image is then exposed to the same imaging conditions as the original (unknown) scene and as represented by the imaging operator $A_{K}$ in equation (1). The result is that the set of LR images $b_{K}{ }^{(1)}$ is generated. Since the image $X_{1}$ is only an approximation of the real scene, these new LR images are different from the original LR images $b_{K}$. The difference between the two sets forms the error vector that should vanish when the reconstructed image converges to the real scene. The error vector is now reconstructed into HR error image, using the interpolation procedure from the previous section, and added to $X_{1}$. The resultant image forms the second approximation $X_{2}$ of the real scene. The image $X_{2}$ is the input for the next iteration. A new set of LR images is generated and subtracted from the original set to form the new error vector, which is reconstructed and added to $\mathrm{X}_{2}$. The resultant image is the third approximation $X_{3}$. The process is repeated until convergence is achieved.

\section{RESULTS}

In order to quantify the fidelity of reconstruction we have again used several known high-resolution images to artificially generate lowerresolution images. The reconstructed HR images were then compared with the original images. A modified version of Root Mean Square Error (RMSE) has been used as a similarity measure. The modification was to ensure that the local spatial contents of images were compared and not their global brightness. The value of RMSE was calculated between the iteratively reconstructed high-resolution image and the original high-resolution image to monitor the reconstruction process.

It is worth mentioning that although the main purpose of the iteration procedure is to minimize artifacts appearing in the interpolated images, the process contains also an implicit regularization features. Both the size of the interpolation kernel as well as the number of iteration strongly affects the smoothness of the reconstructed image and controls the stability of the process. 
Figure 3 shows two examples of the iterative reconstruction of the images from figures 1 and 2 . It is evident that the algorithm successfully removed the artifacts that were visible in the interpolated images, figure 1 and 2 .

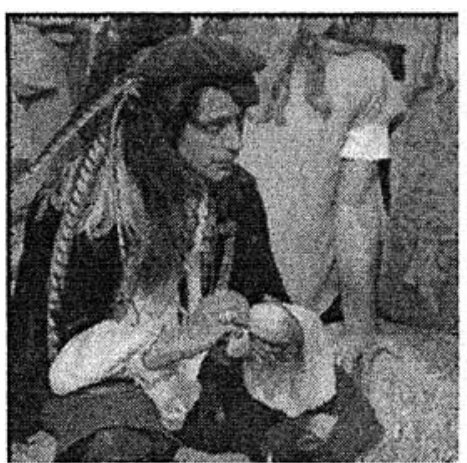

(a)

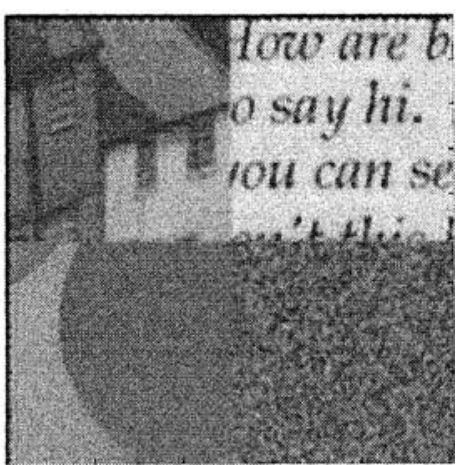

(b)

Figure 3. (a) and (b) - Iteratively Reconstructed High-Resolution Images

A number of different high-resolution test images have been used in the simulation to compare and analyze the performance of the interpolation techniques in generating high-resolution images. For all images the tendencies were similar. Although the RMSE numbers differed for each test image due to the different contents of the images, it has been observed that the most of the improvement occurred during the first few iterations. The further changes in the RMSE values were much smaller and eventually there was no discernible improvement. Typically, as many as 20 iterations were needed to achieve convergence.

The quality of the reconstruction procedure scaled with the number of LR images used in the process. The greater the number of LR images a more densely populated HR grid was generated, and hence the accuracy of the interpolation and reconstruction improved. Note that for larger magnification factors increasing numbers of LR images are necessary to maintain the same level of performance. In most cases, about $10 \mathrm{LR}$ images were sufficient for magnification factors as large as 16. 


\section{SUMMARY AND CONCLUSIONS}

A hybrid reconstruction scheme has been proposed here for application to the problem of super-resolution restoration of high-resolution images from sequences of low-resolution images. The method makes use of interpolation techniques to produce the first approximation for the reconstructed image and then employs an iterative approach to generate the final solution. Preliminary results show that the algorithm is efficient and has a promise of being applicable for real time processing.

Computer experiments were conducted to validate the algorithm. The performance of the method was found to depend significantly on the interpolation method used in reconstruction. Several different interpolation techniques were implemented and tested. As expected, the higher degree interpolation methods were more accurate, leading to better reconstruction. The price for better accuracy, however, was longer computational time. Our results seemed to indicate that the cubic spline interpolation was the most promising in the tradeoff between the accuracy and the computational speed.

The approach presented in this paper requires a relatively small number of low-resolution images for efficient reconstruction. Good results were obtained with only $10 \mathrm{LR}$ frames and for magnification factors as large as 16. This is important for practical applications, because if a large number of LR images were required the accumulation of errors would impede the reconstruction accuracy.

Although the algorithm has been applied to grayscale images only, its extension to RBG images should be straightforward. Further work is also required to test the robustness of the method to noise and registration errors. The extension of the algorithm to more general geometric warping is another future challenge. These issues are currently under investigation.

\section{ACKNOWLEDGEMENTS}

This work is partially supported by Defence Science \& Technology Organisation. Vivek Bannore would like to thank Noel Martin and Lakhmi Jain for supporting this project. Leszek Swierkowski would like to acknowledge valuable discussions with Barnaby Smith. 


\section{REFERENCES}

[1] Super-Resolution Imaging, 1st ed: Kluwer Academic Publishers, 2001.

[2] T. Komatsu, K. Aizawa, T. Igarashi, and T, Saito, "Signal-Processing Based Method For Acquiring Very. High Resolution Images With Multiple Cameras And Its Theoretical Analysis," Communications, Speech and Vision, IEE Proceedings I, vol. 140(1), pp. 19-24, 1993 Feb.

[3] S. C. Park, M. K. Park, and M. G. Kang, "Super-Resolution Inage Reconstruction: A Technical Overview," IEEE Signal Processing Magazine, vol, 20, pp. 21-36, 2003 May.

[4] M. G. Kang and S. Chaudhuri, "Super-Resolution Image Reconstruction," IEEE Signal Processing Magazine, vol 20, pp. 19-20, 2003 May.

[5] M. Elad and A. Feuer, "Restoration of a Single SR Image from Several Blurred, Noisy, \& Under-sampled Measured Images," IEEE Truns. on Image Processing, vol. 6, pp. 1646-1658, 1997 Dec.

[6] W. Zhao and H. Sawhey, "Is Super-Resolution with Optical Flow Feasible?," Proc. ECCV'2002, vol, 1, pp. 599-613, 2002.

[7] M. Elad and A. Feuer, "Super-Resolution Restoration Of An Image Sequence: Adaptive Filtering Approach," Image Processing, IEEE Transactions on, vol. 8(3), pp. $387-395,1999$ March.

[8] R. C. Hardie, J. G. Bognar, K. J, Barnard, and E. A. Watson, "High-Resolution Image Reconstruction from a Sequence of Rotated and Translated Frames and It's Application to an Infrared Imaging System," Optical Engineering, vol. 37, pp. 247 260, 1998 Jan.

[9] M. Irani and S. Peleg, "Improving Resolution by Inage Registration," CVGIP: Graphic Models and Image Processing, vol. 53, pp. 231-239, 1991 May.

[10] T. R. Tuinstra and R. C. Hardie, "High-resolution image reconstruction from digital video by exploitation of non-global motion." Optical Engineering, vol. 38, pp. 806814, 1999 May.

[11] R. Y. Tsai and T. S. Huang, Multiframe Image Restoration and Registration, vol. 1 Greenwich: JAI Press Inc, pp. 317-339, 1984.

[12] N. Nguyen, G. Golub, and P. Milanfar, "Preconditioners for Regularized Image Superresolution," Acoustics, Speech, and Signal Processing. 1999. ICASSP '99. Proceedings., 1999 IEEE International Conference on, vol. 6, pp. 3249 - 3252, 1999 March.

[13] N. Nguyen, P. Milanfar, and G. Golub. "A Computationally Efficient SuperResolution Image Reconstruction Algorthm," Image Processing, IEEE Transactions on, vol. 10(4), pp. 573-583,2001 April.

[14] W. H. Press, B. P. Flannery, S. A. Teukolsky, and W. T. Vetterling, "Iterative Improvement of a Solution to Linear Equations," in Numerical Recipes in C (2nd Edition): Cambridge University Press, 1992 Oct, pp. 1020. 